Article

\title{
Distributed Operation of Microgrids Considering Secondary Frequency Restoration Based on the Diffusion Algorithm
}

\author{
Su-Been Hong ${ }^{1}$, Thai-Thanh Nguyen ${ }^{2}{ }^{\mathbb{D}}$, Jinhong Jeon ${ }^{3}$ and Hak-Man Kim ${ }^{1,2, *(\mathbb{D})}$ \\ 1 Department of Electrical Engineering, Incheon National University, Songdo-dong, 119 Academy-ro, \\ Yeonsu-gu, Incheon 22012, Korea; sbeenhong@gmail.com \\ 2 Research Institute for Northeast Asian Super Grid, Incheon National University, 119 Academy-ro, \\ Yeonsu-gu, Incheon 22012, Korea; ntthanh@inu.ac.kr \\ 3 Division of Smart Distribution Research Center, Korea Electrotechnology Research Institute, \\ Changwon 51543, Korea; jhjeon@keri.re.kr \\ * Correspondence: hmkim@inu.ac.kr; Tel.: +82-32-835-8769; Fax: +82-32-835-0773
}

Received: 24 April 2020; Accepted: 17 June 2020; Published: 20 June 2020

\begin{abstract}
This paper proposes a distributed control of the microgrid (MG) system based on the diffusion algorithm. Unlike the existing decentralized strategy that focuses on the economic operation of the MG system, the proposed strategy performs secondary frequency regulation in addition to the optimization of the MG system. The hierarchical control technique is employed in this study, where the primary layer is responsible for power control and the secondary layer is responsible for the frequency control and economic operation of the MG system. A tested MG system with four distributed generations (DGs) is considered. Three types of communication topologies are evaluated in this study, which are line, ring, and full topologies. The proposed controller is compared to the conventional consensus controller to show the effectiveness of the proposed diffusion controller. Simulation results show that the proposed diffusion strategy improves the convergence speed of the distributed control, resulting in the improvement of power responses and frequency quality of the MG system. The tested system is implemented in the MATLAB/Simulink environment to show the feasibility of the proposed diffusion controller.
\end{abstract}

Keywords: distributed control; diffusion algorithm; distributed operation; frequency control

\section{Introduction}

Microgrid (MG) controllers are commonly based on centralized or decentralized control strategies [1-3]. Centralized controls gather all information of the MG system to find the optimal solution, and are convenient and easy to implement. The reliability of the centralized method is low since all components in the MG system rely on the central controller [4-6]. Decentralized or distributed strategies were proposed to overcome the problem in the centralized control strategies. In the distributed control strategies, every agent is involved in solving the global optimization problem, which reduces the computational burden and improves the reliability of the control system. Easy scalability is an additional advantage of the distributed controls due to the use of a sparse communication network to interchange information, which makes distributed control technology more suitable for MG control [6].

The economic dispatch problem (EDP) is formulated to optimize the operation cost of the MG system, which is easily solved in the central control by the Lagrange multiplier method. It was observed that the condition of a minimum-cost operation for the MG system exists if the incremental costs of all DGs equal an undetermined value [7]. Based on this observation, the economic dispatch problem of 
the MG system is solved in a distributed manner by the incremental cost consensus algorithms [8-10]. Each distributed generator (DG) defines its generation cost function. The incremental cost (IC) value of such cost function is calculated according to power generation of each DG. Then, the information of IC value is exchanged with the neighbor DGs. The optimal solution of the EDP is found if all IC values reach a common value that satisfies the power balance constraint. These strategies were also applied to the multi-microgrid system [11].

The communication network is utilized for information exchange between DGs in the MG system. The optimal solution is found after a number of iterations of information exchange. Thus, the convergence speed of the distributed algorithm affects to the performance of the MG operation. The diffusion strategies were adopted to improve the convergence properties of the distributed control [12-15]. These strategies require the load forecasting algorithm to satisfy the power balance constraint and obtain the optimal solution of the MG operation. However, the uncertainties in load forecasting algorithm negatively affects to the optimal solution of the MG operation. A two-step diffusion algorithm in $[16,17]$ was proposed to overcome the problem, in which the total load of the MG system is found in the first step, and the EDP optimization is solved in the second step. However, the accuracy of load prediction in the first step relies on the local controller of each DG. Since the MG system is controlled by the droop controller, the mismatch between total demand and total power generation exists, which leads to inaccuracy of the optimal solution found in the second step. The problem could be improved by employing the secondary frequency control schemes.

Various studies on the secondary frequency regulation have been proposed to overcome the problem in the droop controllers [18-21]. The distributed controllers using consensus algorithms for the frequency regulation have also been presented [22-24]. However, these studies focused only on the frequency regulation of the MG system, while the economic problem of the MG system was not considered. An additional control layer is required to optimize the operation of the MG system, which results in an increase in the complexity and cost of the MG system. It is well-known that the power balance between demand and generation is achieved when the system frequency is maintained at the nominal value. Based on this observation, this study proposes a distributed control strategy of the MG system involving the optimization of both economic and frequency deviation of the MG system. With the proposed controller, the power balance constraint is maintained by the minimization of the frequency deviation, avoiding the load prediction step in existing distributed operation algorithms. Thus, the proposed control strategy would maintain both economic operation and secondary frequency regulation of the MG system. The main contributions of this study are as follows:

- A distributed control strategy of the MG system is proposed, which maintains both economic operation and frequency restoration of the MG system in a single layer. The proposed strategy avoids the load prediction step by employing the optimization of frequency deviation, which overcomes the problem of load uncertainty in the optimization problem.

- A diffusion-based distributed control is proposed to improve the convergence speed of the distributed control, leading to an improvement in power response and frequency regulation.

The proposed method is based on the hierarchical strategy with two layers, in which the secondary layer is responsible for the economic operation of the MG system and the secondary frequency regulation. The typical droop controllers are used in the local controller to achieve initial power sharing among DGs. Then, the secondary controller based on the diffusion algorithm calculates the additional power of each DGs to optimize the economic operation of the MG system. The MG system consists of four DGs used to verify the proposed method. The effectiveness of the proposed diffusion-based MG controller is evaluated by testing the proposed method with three communication topologies-line, ring, and full connections. In addition, a comparison study on the proposed diffusion-based MG controller and the conventional consensus-based controller is presented in this study.

The paper organization is as follows. The hierarchical control techniques of the MG system is described in Section 2. The proposed distributed operation of the MG system based on the diffusion 
algorithm is presented in Section 3. Simulation results are provided in Section 4. Finally, the main conclusion is summarized in Section 5.

\section{Control Strategies of the MG System}

The hierarchical control of the MG system shown in Figure 1a consists of three layers, which are the primary layer, secondary layer, and tertiary layer. The primary layer is the local controller of each DG and it requires only local DG information. The primary layer is responsible for local voltage control and proper power sharing between multiple DGs. Since the rotating inertia of the MG system is low due to the lack of synchronous generators, the primary controller must be fast (i.e., operating in milliseconds) to ensure stability during operations of the MG system. Furthermore, in order to enhance the reliability of the MG, the communication system could be neglected in the primary control. The secondary and tertiary control layers support the MG operation with multiple objectives. A low-bandwidth communication link is often used in the secondary layer to achieve the global controllability. It could be based on the centralized or decentralized strategies with the objective of frequency of voltage restoration or other objectives related to the voltage control and power qualities.

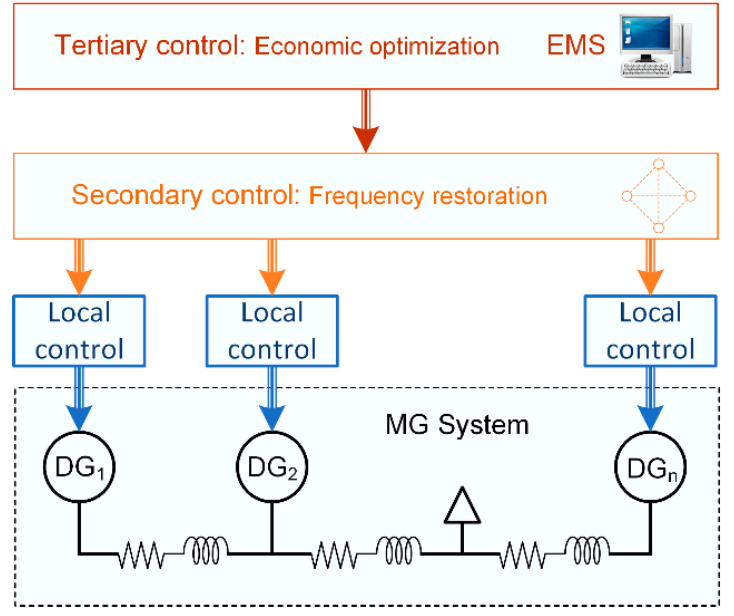

(a)

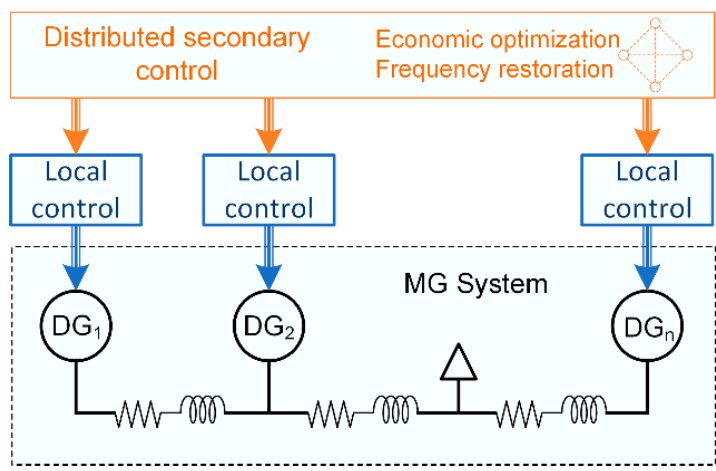

(b)

Figure 1. Microgrid control strategies: (a) conventional hierarchical control; (b) proposed distributed control.

In the MG system, the tertiary layer could be utilized for economic optimization considering the electricity markets and cost of power. The tertiary control layer in the conventional hierarchical control is commonly based on the centralized methods, which offer the benefit of easy implementation. However, the central controller suffers the computation burden when the system scale significantly increases. In addition, the reliability of the control system is low since all DGs in the MG system rely on a single central controller.

The problem is overcome by the proposed distributed controller shown in Figure $1 \mathrm{~b}$. The secondary control layer in the proposed strategy is responsible for both economic operation and frequency restoration of the MG system. The sparse communication network is used to interchange information among DGs. All DGs are coordinated to optimize the economic problem of the MG system, which results in a reduction in computation burden and an improvement in the reliability of the control system. It is well-know that the power balance is achieved when the system frequency is regulated at the nominal value. The distributed optimization in the proposed strategy is subjected to the constraint of frequency deviation, which results in the frequency restoration of the MG system.

The flow diagram of the proposed distributed controller is shown in Figure 2. Optimal power indicates that the minimizing of the operation cost and frequency deviation is calculated in the secondary layer by the diffusion algorithm. Each DG receives output power information from its 
neighbors $\left(P_{j}\right)$ and locally estimates the system frequency by the phase-locked loop (PLL). Based on this information, the diffusion algorithm is used to calculate the incremental cost of each DG, and the optimal power reference $\left(P_{i}^{*}\right)$ is found accordingly. The optimal power reference is sent to the local controller where the conventional droop power controller is employed to manage the output power of each DG. Finally, the output power of each DG is measured and broadcasted to its neighbors. Iterating this process leads to the convergence of all incremental costs to a common value, resulting in the optimal condition of the MG system. The local control and secondary control layers in the proposed strategy are explained in next section.

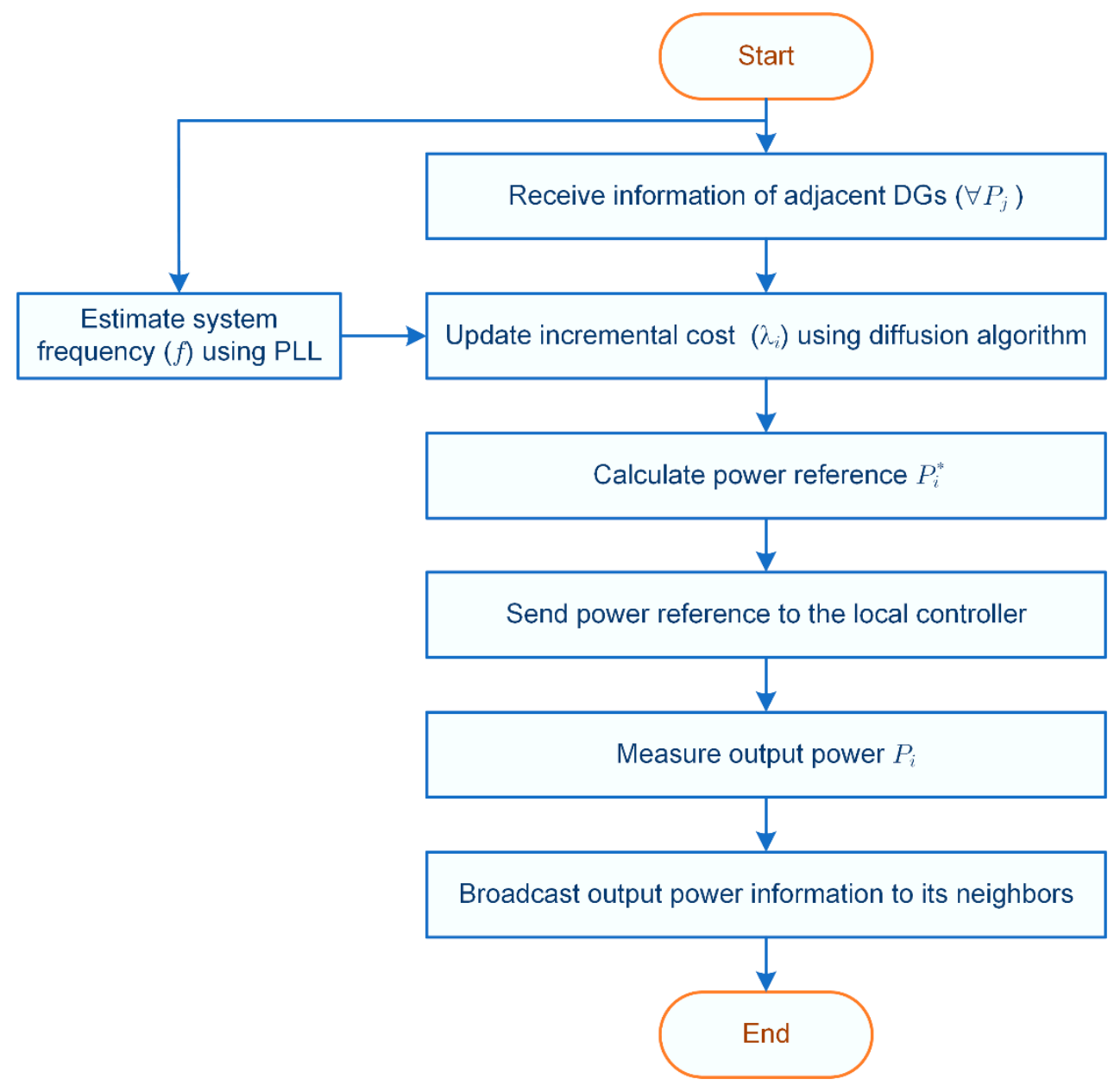

Figure 2. Flow diagram of proposed controller.

\section{Proposed Distributed MG Control}

The MG system could include various types of distributed generations (DGs), such as photovoltaic, fuel cells, and wind generations. Such DGs are interfaced with the MG system by the power converters to improve aspects of their controllability, such as power or voltage regulation. These configurations of such DGs are known as inverter-based DGs. A typical configuration of an inverter-based DG is shown in Figure 3, which is based on the two-level voltage source converter with an output LC filter connected with a constant DC source. The proposed controller for each DG consists of two layers: a primary and secondary, where the primary layer is responsible for power control and the secondary layer is in charge of frequency restoration and economic optimization. 


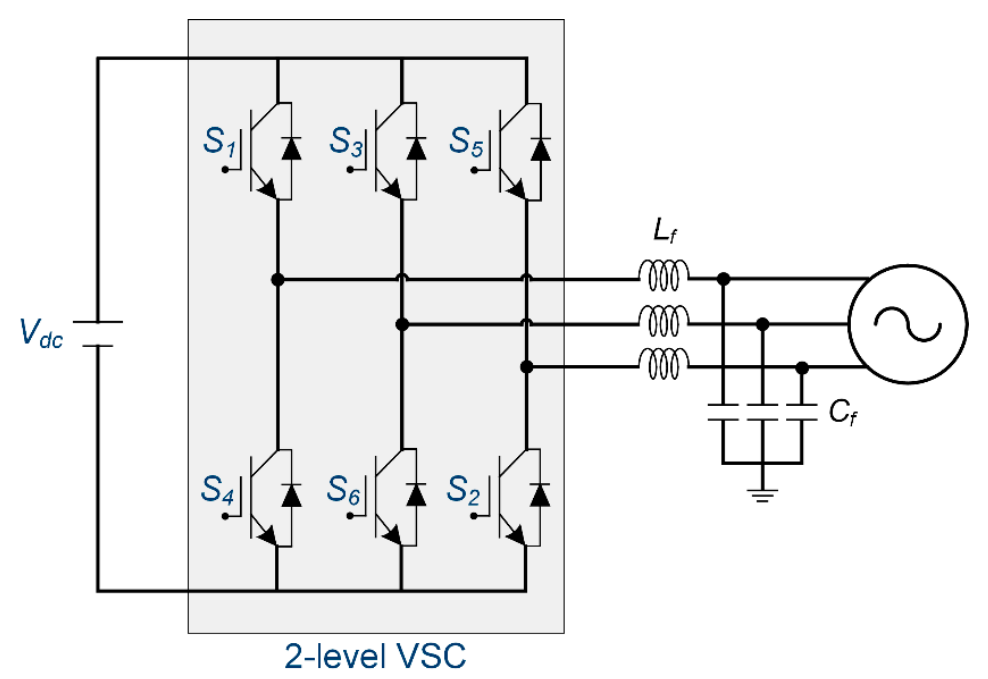

Figure 3. Distributed generator based on two-level voltage source converter.

\subsection{Primary Control of DGs}

The primary control of DG is shown in Figure 4, which includes the inner current/voltage control and outer power control loops. The proportional integral (PI) regulators are used to regulate the converter current and voltage. The current controller is based on the decoupling of a dq-reference frame using Equations (1) and (2) [25].

$$
\begin{aligned}
& v_{g d}^{*}=k_{P 1}\left(i_{g d}^{*}-i_{g d}\right)+\int k_{I 1}\left(i_{g d}^{*}-i_{g d}\right) d t-\omega_{g} L_{f} i_{g q} \\
& v_{g q}^{*}=k_{P 2}\left(i_{g q}^{*}-i_{g q}\right)+\int k_{I 2}\left(i_{g q}^{*}-i_{g q}\right) d t-\omega_{g} L_{f} i_{g d}
\end{aligned}
$$

where $k_{P 1}$ and $k_{I 1}$ are PI control coefficients of the inner current control loop of $d$ axes, $k_{P 2}$ and $k_{I 2}$ are PI control coefficients of the inner current control loop of $q$ axes, $i_{g d}^{*}$ and $i_{g q}^{*}$ are the stator currents of the $i_{g d}$ and $i_{g q}$ reference of the $d$ and $q$ axes, and $v_{g d}^{*}$ and $v_{g q}^{*}$ are the output voltage references.

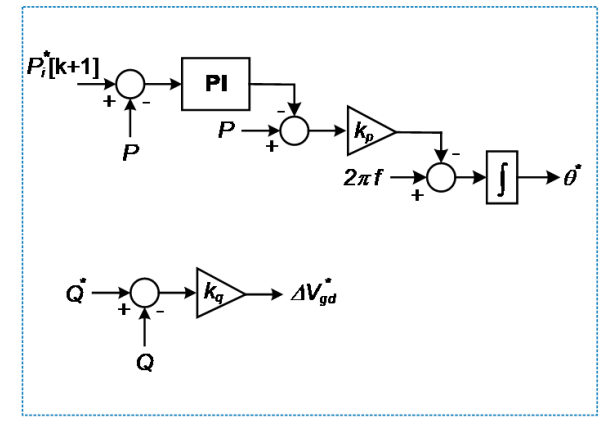

Droop power control

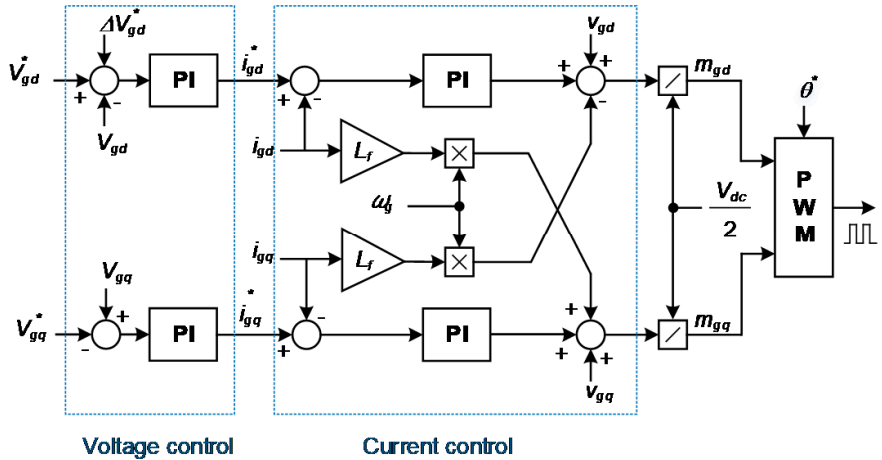

Voltage control

Current control

Figure 4. Local controller of distributed generators.

The outer control loop is based on the droop control mechanism to manage the power sharing between multiple DGs in the MG system. The droop controller is used to share load power demand through proportional constants and ensure angular frequency/voltage stability. The droop control loop generates the references of angular frequency and voltage to the inner voltage control loop, as given by Equations (3) and (4).

$$
\theta^{*}=\int\left\{2 \pi f-k_{p}\left(P-\Delta P^{*}\right)\right\} d t
$$




$$
\Delta V_{g d}^{*}=-k_{q}\left(Q^{*}-Q\right)
$$

where $\theta^{*}$ and $\Delta V_{g d}^{*}$ are the angular frequency and amplitude deviation of voltage, $f$ is the nominal frequency, and $k_{p}$ and $k_{q}$ are $\mathrm{v}$, respectively. $Q^{*}$ and $\Delta P^{*}$ are the reference values for the DG's reactive power and deviation of active power, respectively, and $P$ and $Q$ and represent the measurements. Thus, the angular frequency is dependent on active power and the difference in voltage amplitude is dependent on reactive power.

\subsection{Diffusion-Based Secondary Control of the Microgrid System}

\subsubsection{Optimal Operation of the MG System}

It is assumed that there are $n$ distributed generators in the MG system. The output power of $i$-th $\mathrm{DG}_{\mathrm{i}}$ is equal to $P_{i}$, and $P_{D}$ is the load demand of the MG system. The cost function for each DG can be expressed by the quadratic function $C_{i}\left(P_{i}\right)$, as shown in Equation (5) [26].

$$
C_{i}\left(P_{i}\right)=\alpha_{i} P_{i}^{2}+\beta_{i} P_{i}+\gamma_{i}
$$

where $\alpha_{i}, \beta_{i}$ and $\gamma_{i}$ are cost coefficients.

The optimal output powers of DGs are found by minimizing the objective function $C$ in Equation (6).

$$
C=\sum_{i=1}^{n} C_{i}\left(P_{i}\right)
$$

Subject to the constraint's power balance and power capacity.

$$
\begin{gathered}
P_{D}=\sum_{i=1}^{n} P_{i} \\
P_{i, \text { min }} \leq P_{i} \leq P_{i, \text { max }}
\end{gathered}
$$

The Lagrange multiplier method is applied to solve the objective function $C$. The Lagrange function $\mathcal{L}$ is given by the objective function $C$ and the constraint function multiplied by the undetermined multiplier $\lambda$, as shown in Equation (9).

$$
\mathcal{L}=\sum_{i=1}^{n} C_{i}\left(P_{i}\right)+\lambda\left(P_{D}-\sum_{i=1}^{n} P_{i}\right)
$$

Considering the derivative of $\mathcal{L}$ with respect to each variable, we have:

$$
\begin{aligned}
& \frac{\partial \mathcal{L}}{\partial P_{i}}=\frac{\partial C_{i}\left(P_{i}\right)}{\partial P_{i}}-\lambda \\
& \frac{\partial \mathcal{L}}{\partial \lambda}=P_{D}-\sum_{i=1}^{n} P_{i}
\end{aligned}
$$

Setting the derivatives of Equations (10) and (11) to 0 gives the optimal solution. Thus, the cost operation of the MG system reaches to minimum when the IC of each DG is equal to $\lambda$, as given by Equation (12) [27].

$$
\lambda=\frac{P_{D}+\sum_{i=1}^{n} \frac{\beta_{i}}{2 \alpha_{i}}}{\sum_{i=1}^{n} \frac{1}{2 \alpha_{i}}}
$$


Thus, the optimal power of each DG is given by Equation (13).

$$
P_{i}^{*}=\frac{\lambda-\beta_{i}}{2 \alpha_{i}}
$$

\subsubsection{Proposed Diffusion-Based Distributed Control of the MG System}

It can be observed in previous section that the optimal operation of the MG system is maintained if the incremental cost of each DG in the MG system converges to a common value. In the secondary control layer, the communication network is utilized to inform the neighbor-to-neighbor information trade and to find the optimal value of the incremental cost. In the mechanism, each DG will send their respective output powers $P_{i}$ to neighborhoods, and receive information from those neighborhoods with $\mathrm{DG}_{\mathrm{j}}\left(P_{j}\right)$ with $j \in N_{i}$, where $N_{i}$ is the neighborhood of $\mathrm{DG}_{\mathrm{i}}$. The undirected graph $\mathrm{G}_{N}=\left(\mathrm{N}_{N}, \mathrm{E}_{N}\right)$, with the set of nodes $\mathrm{N}_{N}=\left\{n_{1}, n_{2}, \ldots, n_{N}\right\}$ representing the $N$ controllers of DGs, and the communication links between controllers represented by the set of edges $\mathrm{E}_{N} \subseteq \mathrm{N}_{N} \times \mathrm{N}_{N}$ express the communication network established between the DGs. The edge $\left(n_{i}, n_{j}\right)$ in the aforementioned edge set $\mathrm{E}_{N}$ shows that node $\mathrm{n}$ receives information from node $n_{j}$. The undirected graph $\mathrm{G}_{N}$ defined as as $\mathrm{A}_{N}=\left[a_{i j}\right] \in \mathbb{R}^{N \times N}$ is also associated with the adjacency matrix, where $a_{i j}$ is a positive value supposing that if $\left(n_{i}, n_{j}\right) \in \mathrm{E}_{N}$ and $a_{i j}=0$ otherwise. The Laplacian matrix of the undirected graph $\mathrm{G}_{N}$ is noted as $\mathrm{L}_{N}=\left[l_{i j}\right] \in \mathbb{R}^{N \times N}$, where $l_{i i}=\sum_{j=1, j \neq i}^{N} a_{i j}$ and $l_{i j}=-a_{i j}$ is for $j \neq i$. The global dynamic of the combine-then-adapt diffusion strategy is as shown in Equation (14) [28],

$$
\left\{\begin{array}{c}
\phi[\mathrm{k}]=(\mathrm{I}-\varepsilon \mathrm{L}) \mathrm{X}[\mathrm{k}] \\
\mathrm{X}[\mathrm{k}+1]=\phi[\mathrm{k}]-\mu \nabla \phi[\mathrm{k}]
\end{array}\right.
$$

where $X_{k}=\left[x_{1, k} ; x_{2, k} ; \ldots ; x_{N, k}\right]$ indicates each state of DGs during time $k ; \phi[\mathrm{k}]$ is the intermediate variables of DGs at time $k ; \varepsilon$ is the weight of the constant edge; $\mu \in \mathbb{R}^{+}$indicates the updating parameter; $\nabla \phi[\mathrm{k}]$ is assumed to be the stochastic gradient of variables $\phi[\mathrm{k}]$.

The diffusion algorithm in Equation (14) is used in each DG to find the optimal incremental cost. The secondary control objective of frequency is added in the diffusion algorithm to improve the frequency of the MG system. Unlike the conventional distributed operation strategy, the constraint of power balance is maintained by regulating the system frequency at the nominal value. The proposed diffusion algorithm is given by the following:

$$
\left\{\begin{array}{c}
\phi[\mathrm{k}]=(\mathrm{I}-\varepsilon \mathrm{L}) \lambda[\mathrm{k}] \\
\lambda[\mathrm{k}+1]=\phi[\mathrm{k}]-\mu \nabla \phi[\mathrm{k}]-\eta\left(f^{*}-f\right)
\end{array}\right.
$$

where $\lambda[\mathrm{k}]=\left[\lambda[\mathrm{k}]_{1} ; \lambda[\mathrm{k}]_{2} ; \ldots ; \lambda[\mathrm{k}]_{N}\right]$ are the incremental cost of a DG at time $k ; f$ is the measured system frequency; and $f^{*}$ is the nominal frequency of the MG system.

The optimal power of each DG is found according to the corresponding incremental cost, as given by

$$
P_{i}^{*}[\mathrm{k}+1]=\frac{\lambda[\mathrm{k}+1]-\beta_{i}}{2 \alpha_{i}}
$$

In the secondary diffusion control, the control block diagram is supposed as Figure 5, where the inputs are the power information of the adjacent DGs and the output is the optimal power reference for the local controller. 


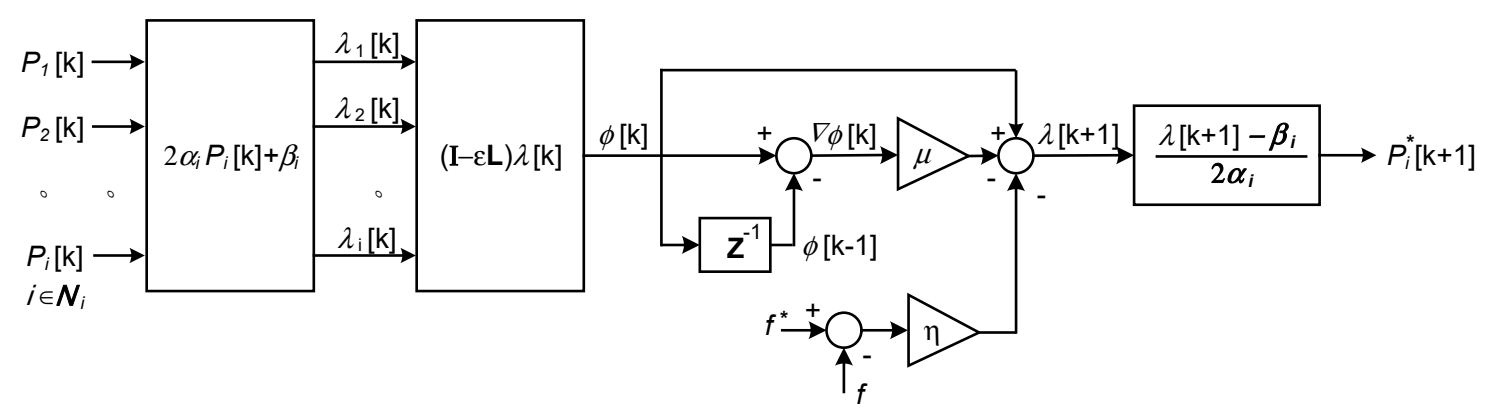

Figure 5. Secondary diffusion controller considering frequency restoration.

\section{Simulation Results}

In this study, the tested MG system is illustrated in Figure 6, consisting of four DGs and three loads. The distribution lines are modelled by the resistance/inductance components. Parameters of the DGs and the tested MG system are shown in Tables 1 and 2. Table 3 shows the converter parameters used in this study. Since this study focuses on the economic operation and secondary frequency regulation of the MG system, the converter modelling is based on the average model. The designs of the PI gains of current and voltage control are based on the pole-placement method, as discussed in [29]. The three communication topologies (line, ring and full topology) of this study are given in Figure 7. In the MATLAB/Simulink environment, the communication network is modelled using From/Goto blocks, in which the Goto block is used to transmit information and the From block is used to receive information. The scenario of step load change is used to verify the performance of the proposed control strategy. It is assumed that the initial load is $8 \mathrm{~kW}$ and an additional load of $4 \mathrm{~kW}$ is connected to the MG system at $5 \mathrm{~s}$.

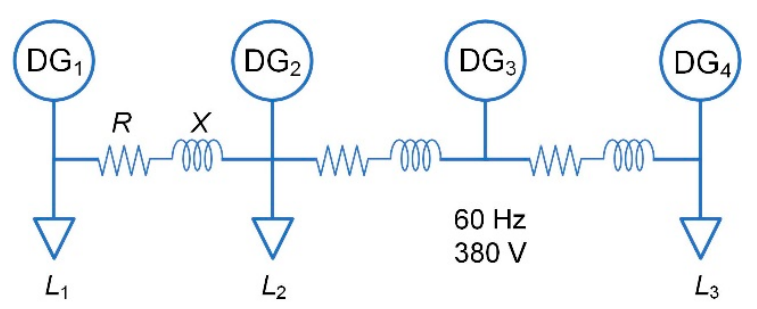

Figure 6. Tested MG system with four DGs.

Table 1. System parameters.

\begin{tabular}{ccc}
\hline Parameter & Symbol & Value \\
\hline Line Resistance & $R$ & $0.0355 \Omega$ \\
Line Inductance & $X$ & $0.15 \mathrm{mH}$ \\
Load 1 & $L_{1}$ & $3 \mathrm{~kW}$ \\
Load 2 & $L_{2}$ & $4 \mathrm{~kW}$ \\
Load 3 & $L_{3}$ & $5 \mathrm{~kW}$ \\
System frequency & $f$ & $60 \mathrm{~Hz}$ \\
System voltage & $V_{g}$ & $380 \mathrm{~V}$ \\
\hline
\end{tabular}

Table 2. Parameters of generation cost of each DG.

\begin{tabular}{cccc}
\hline Unit & $\boldsymbol{\alpha}_{\boldsymbol{i}}\left(\mathrm{USD} / \mathbf{k W}^{2} \mathbf{h}\right)$ & $\boldsymbol{\beta}_{\boldsymbol{i}}(\mathrm{USD} / \mathbf{k W h})$ & $\gamma_{\boldsymbol{i}}(\mathrm{USD} / \mathbf{k W h})$ \\
\hline 1 & 0.001562 & 3.92 & 561 \\
2 & 0.00194 & 3.85 & 310 \\
3 & 0.00482 & 3.97 & 78 \\
4 & 0.00252 & 3.75 & 250 \\
\hline
\end{tabular}


Table 3. Converter parameters.

\begin{tabular}{ccc}
\hline Parameter & Symbol & Value \\
\hline Filter Inductance & $L_{f}$ & $2.5 \mathrm{mH}$ \\
Filter Capacitance & $C_{f}$ & $35 \mu \mathrm{F}$ \\
Output AC voltage & $v_{g}$ & $380 \mathrm{~V}$ \\
DC-link voltage & $\mathrm{V}_{\mathrm{dc}}$ & $800 \mathrm{~V}$ \\
PI gains of current control & $k_{P} / k_{i}$ & $10.5 / 16,000$ \\
PI gains of outer voltage & $k_{P} / k_{i}$ & $0.05 / 390$ \\
control & $k_{p} / k_{q}$ & $9.4 \times 10^{-4} / 1.94 \times 10^{-3}$ \\
Droop gains & $T_{s}$ & $50 \mu \mathrm{s}$ \\
Sampling time & $T_{c o m}$ & $0.1 \mathrm{~s}$ \\
\hline Communication time & &
\end{tabular}

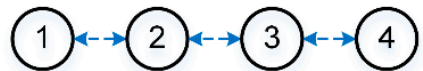

(a)

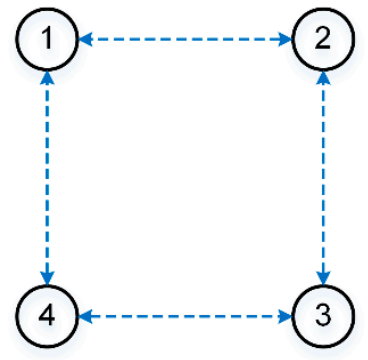

(b)

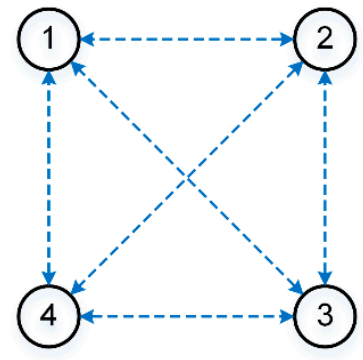

(c)

Figure 7. Communication topologies: (a) line; (b) ring; (c) full.

When solving the incremental cost (IC) of each DG using the Lagrange multiplier method, the optimal IC $\left(\lambda^{*}\right)$ with a power demand of $12 \mathrm{~kW}$ is $17.5 \mathrm{USD} / \mathrm{kW}$. The corresponding powers of the four DGs are $4.35 \mathrm{~kW}, 3.52 \mathrm{~kW}, 1.4 \mathrm{~kW}$, and $2.73 \mathrm{~kW}$, respectively. Figures 8-10 show the optimal IC found by the distributed controllers, and it is consistent with the Lagrange multiplier method. The optimal IC found by the distributed control is $17.5 \mathrm{USD} / \mathrm{kW}$ after several iterations. The convergence of ICs depends on the communication topologies and the control methods. The optimal IC is found after $1 \mathrm{~s}$ with the full connection, whereas it takes more than $5 \mathrm{~s}$ in the case of line connection. The advantage of the proposed distributed control based on the diffusion algorithm can be seen in Figure 8, which shows a faster convergence speed compared to the conventional consensus method. The optimal IC is found at $8.9 \mathrm{~s}$, whereas it is found at more than $5 \mathrm{~s}$ in the case of the conventional controller.
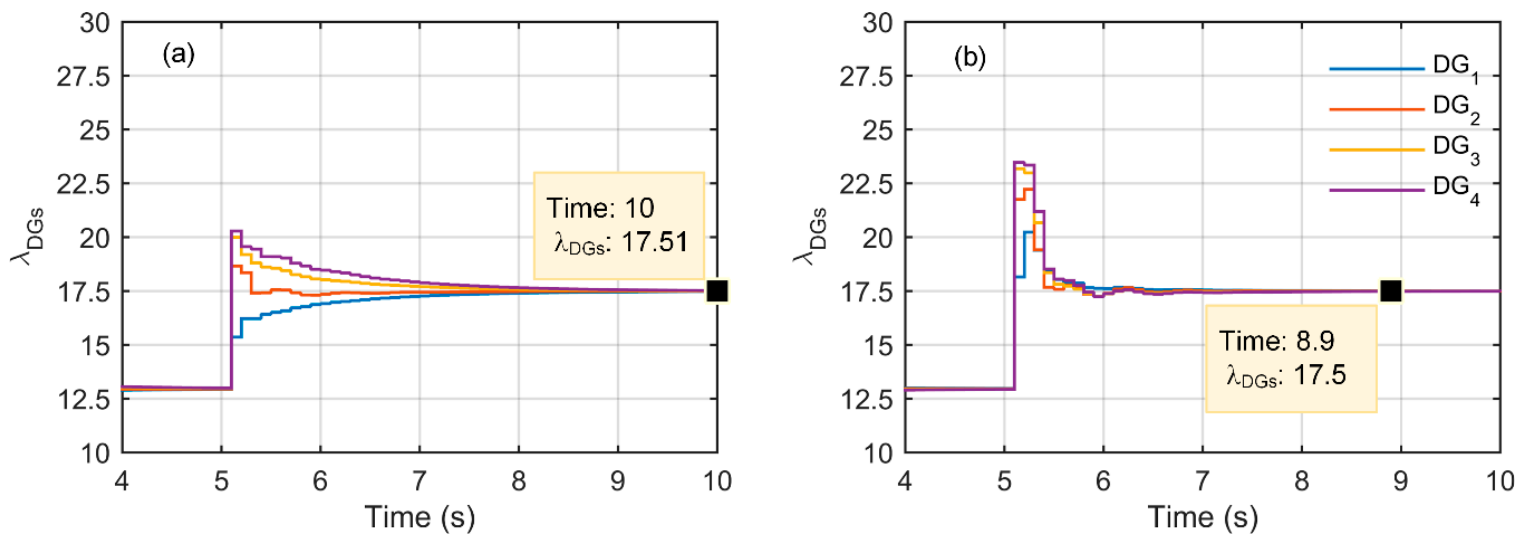

Figure 8. Lambda in the case of line connection: (a) conventional consensus-based control; (b) proposed diffusion control. 

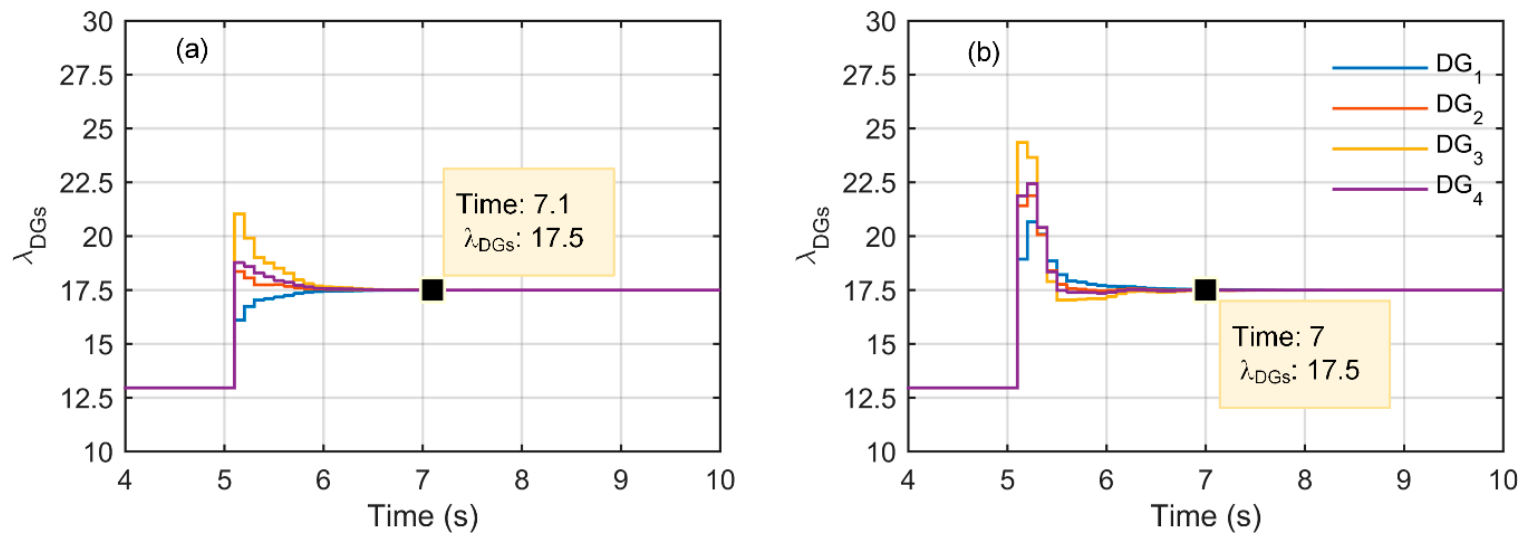

Figure 9. Lambda in the case of ring connection: (a) conventional consensus-based control; (b) proposed diffusion control.
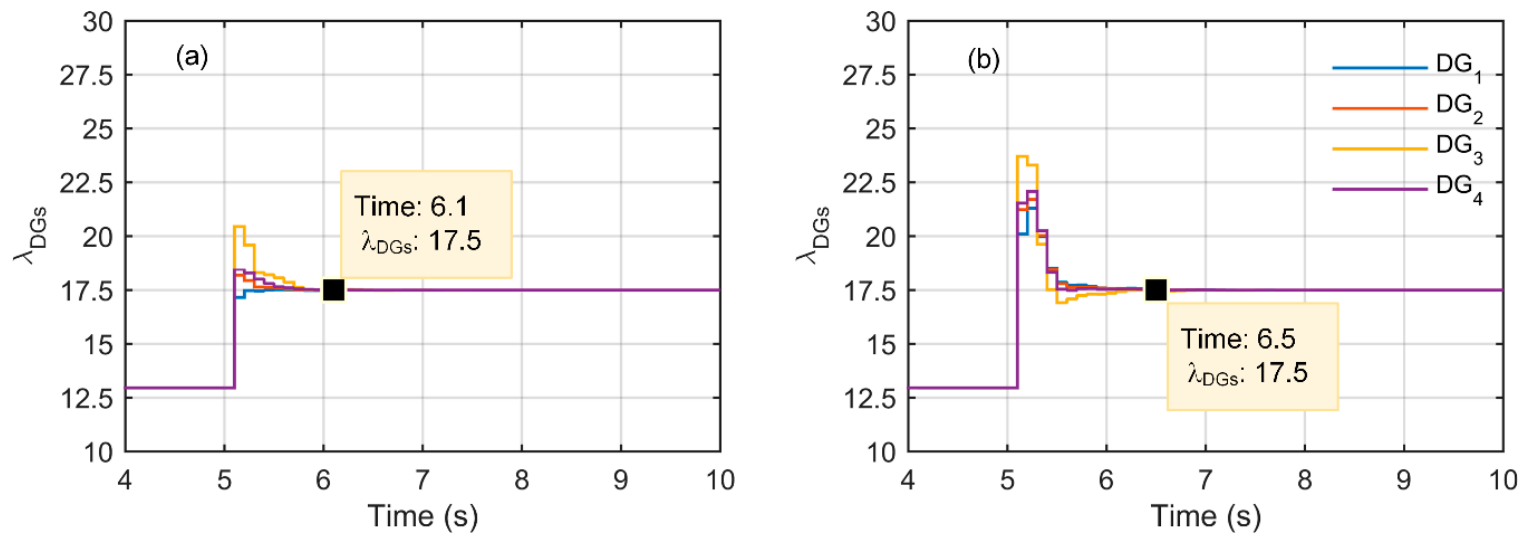

Figure 10. Lambda in the case of full connection: (a) conventional consensus-based control; (b) proposed diffusion control.

The convergence speed of the ICs has an impact on the response of the output powers, which is represented in Figures 11-13. Load changing at $5 \mathrm{~s}$ causes the transient output power. The steady-state output powers of DGs shown in these figures are consistent with the Lagrange multiplier method. It can be observed that fast convergence speed results in a fast response of the output powers. The communication topology of full connection provides the fastest power responses compared to the line and ring connections. With the proposed diffusion-based controller, the responses of output powers are much quicker than the conventional consensus method, particularly in the case of the line connection.

The system frequency under the two distributed controllers is shown in Figure 14. With the conventional consensus controller, there is an existence of a frequency drop below the nominal frequency. When the load is increased, the frequency drops to $59.9 \mathrm{~Hz}$. However, with the proposed diffusion controller, the frequency is maintained stably at $60 \mathrm{~Hz}$. When additional load connects to the MG system, the system frequency temporarily shows a slight reduction, and then it gradually recovers to the recorded value of $5.58 \mathrm{~s}$. 

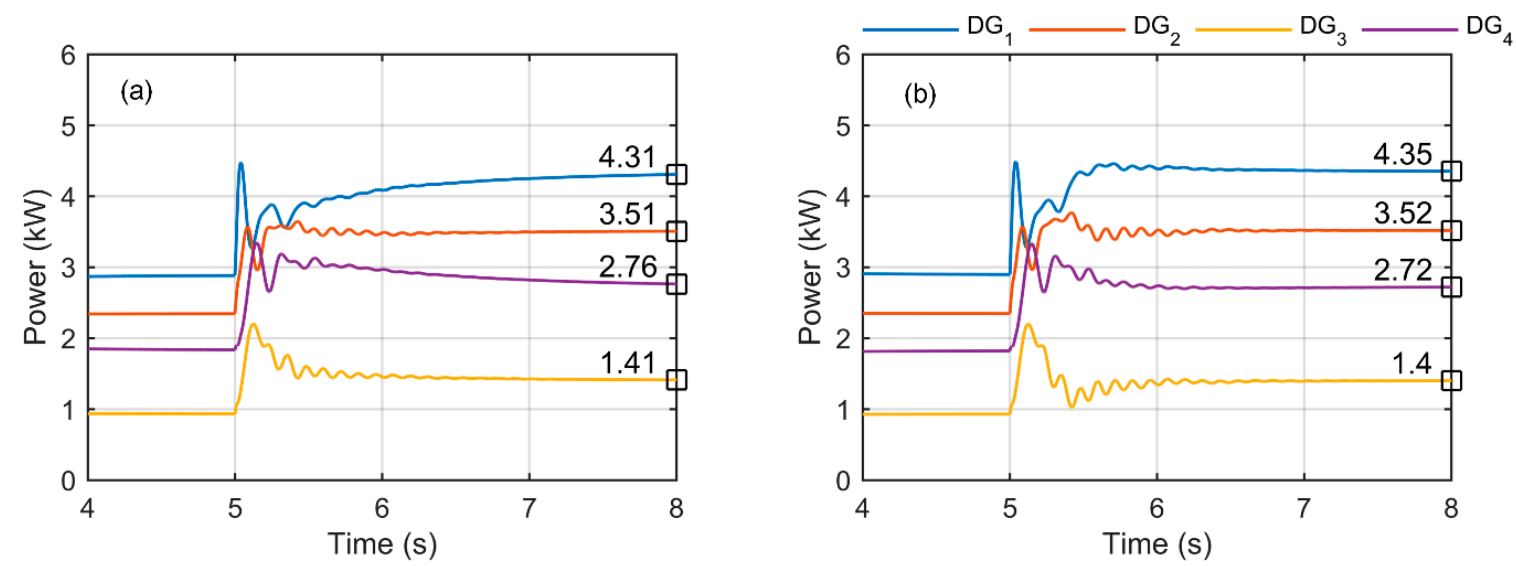

Figure 11. Output power of DGs in the case of line connection: (a) conventional consensus-based control; (b) proposed diffusion control.
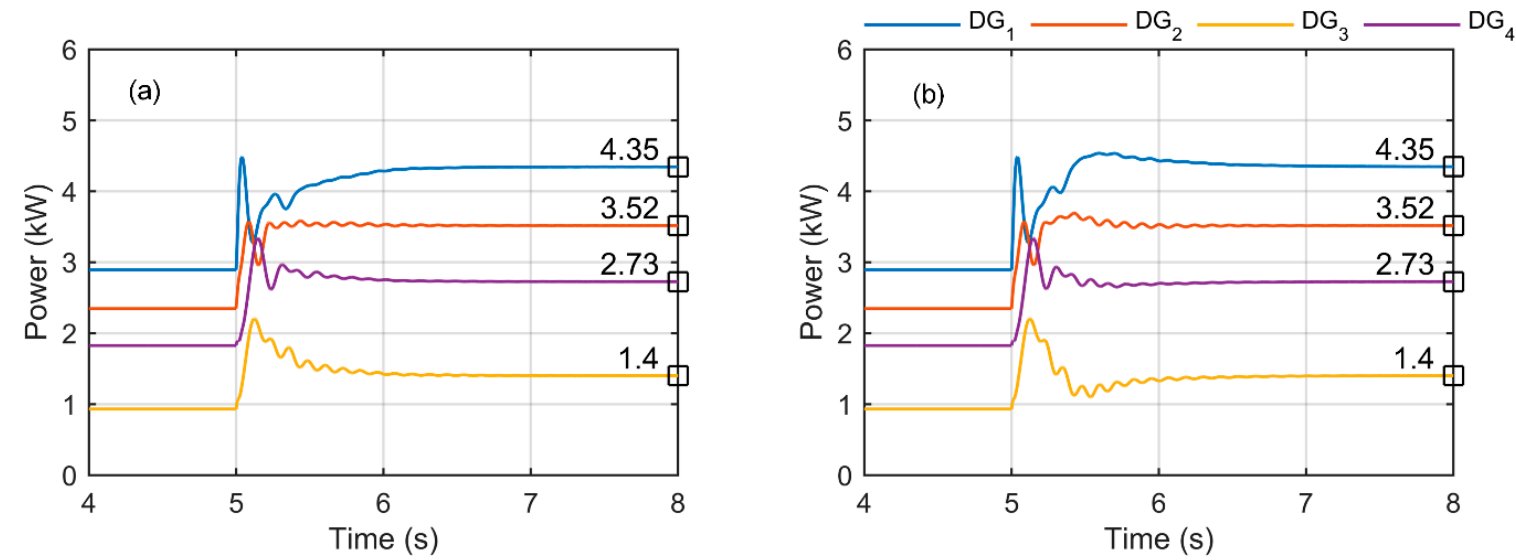

Figure 12. Output power of DGs in the case of line connection: (a) conventional consensus-based control; (b) proposed diffusion control.
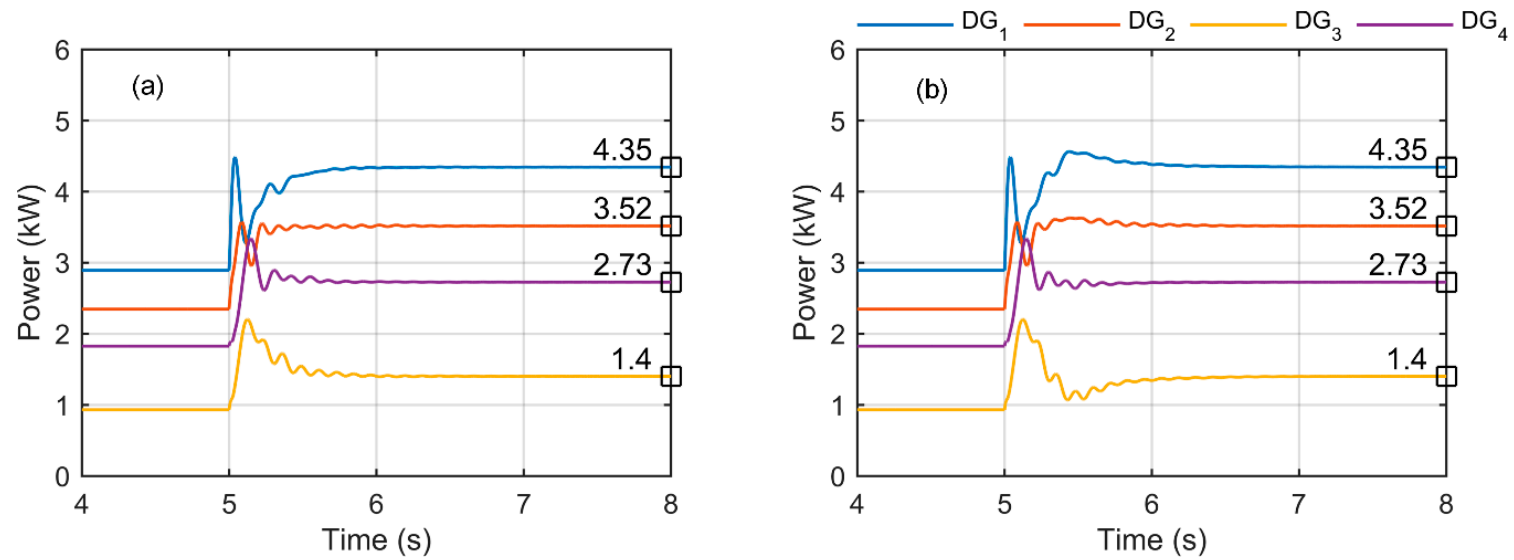

Figure 13. Output power of DGs in the case of line connection: (a) conventional consensus-based control; (b) proposed diffusion control. 

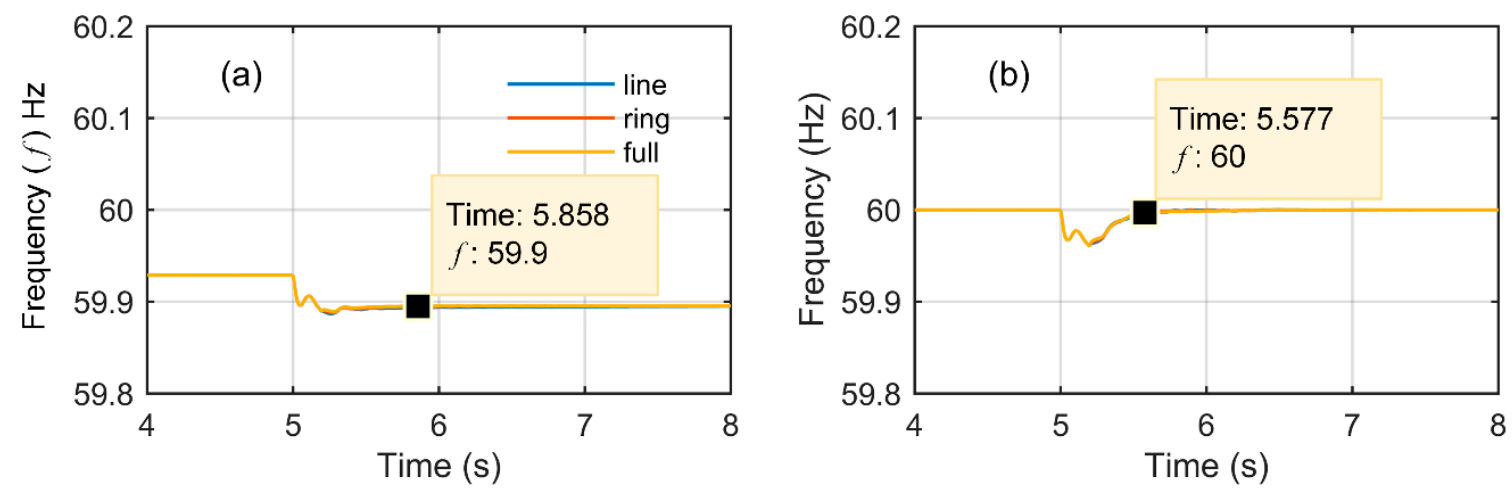

Figure 14. System frequency: (a) conventional consensus-based control; (b) proposed diffusion control.

Figure 15 shows the cost comparison study with the secondary frequency control schemes in $[30,31]$. With the proposed control strategies in [30,31], all DGs equally supply power to restore the system frequency, which leads to a higher operation cost of the MG system. By comparison, the proposed strategy manages different power generations among DGs to minimize the operation cost while restoring the system frequency to the nominal value. It can be seen that the operation cost of the MG system with the proposed diffusion controller is smaller than that of the existing controller in $[30,31]$.

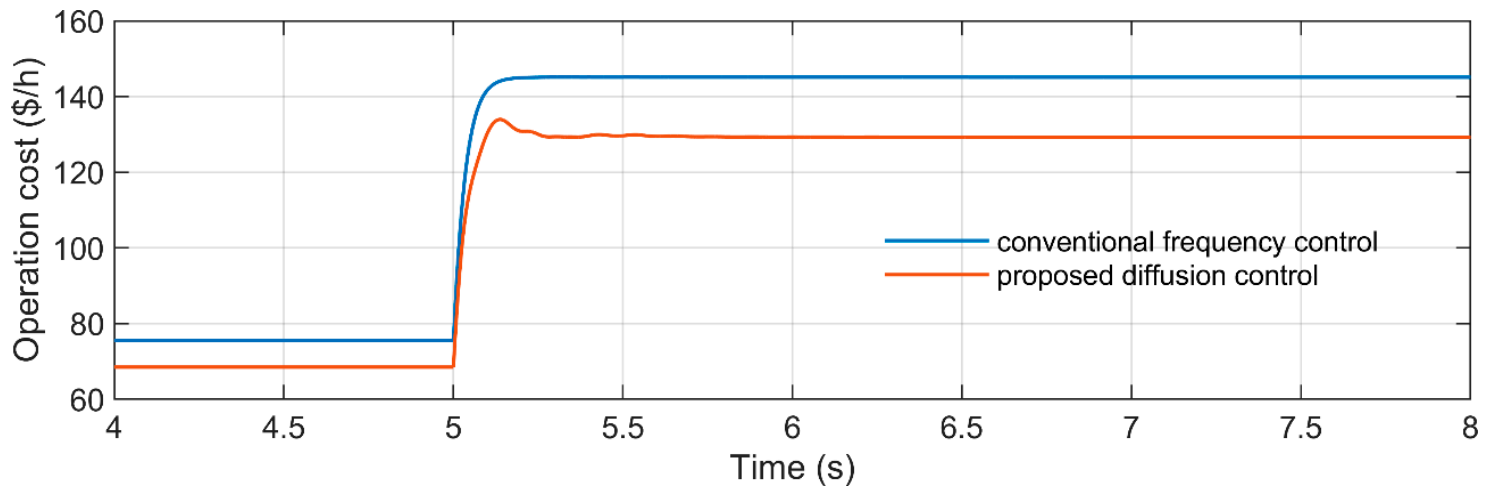

Figure 15. Comparison of operation costs.

\section{Conclusions}

This paper has proposed a distributed control of the MG system based on the diffusion strategy. The proposed controller not only optimized the economic operation of the MG system but also maintained the system frequency at the nominal value. By considering the secondary frequency regulation, the load prediction could be avoided in the proposed distributed control since the power balance constraint is kept by regulating the system frequency. The proposed method is in the form of a diffusion algorithm added to the existing primary control system. The proposed strategy was tested in three communication topologies and was compared to the conventional method. The proposed diffusion-based controller showed the advantage of a fast convergence speed compared to the conventional consensus method, particularly in the case of line connection, which has a positive impact on the power response of DGs. The proposed method can be considered as a promising solution for the distributed operation of the MG system.

Author Contributions: Conceptualization, investigation, and writing-original draft preparation, S.-B.H.; writing-review, visualization and editing, T.-T.N.; funding acquisition and project administration, J.J. writing-review and editing and supervision, H.-M.K. All authors have read and agreed to the published version of the manuscript. 
Funding: This research was supported by the Korea Electrotechnology Research Institute (KERI) Primary Research Program through the National Research Council of Science \& Technology (NST), funded by the Ministry of Science and ICT (MSIT) (No. 20A01056).

Conflicts of Interest: The authors declare no conflict of interest.

\section{References}

1. Zhao, J.; Dörfler, F. Distributed control and optimization in DC microgrids. Automatica 2015, 61, 18-26. [CrossRef]

2. Bolognani, S.; Zampieri, S. A distributed control strategy for reactive power compensation in smart microgrids. IEEE Trans. Automat. Contr. 2013, 58, 2818-2833. [CrossRef]

3. Morstyn, T.; Hredzak, B.; Demetriades, G.D.; Agelidis, V.G. Unified distributed control for DC microgrid operating modes. IEEE Trans. Power Syst. 2016, 31, 802-812. [CrossRef]

4. Anand, S.; Fernandes, B.G.; Guerrero, J. Distributed control to ensure proportional load sharing and improve voltage regulation in low-voltage DC microgrids. IEEE Trans. Power Electron. 2013, 28, 1900-1913. [CrossRef]

5. Liu, W.; Zhuang, P.; Liang, H.; Peng, J.; Huang, Z. Distributed Economic Dispatch in Microgrids Based on Cooperative Reinforcement Learning. IEEE Trans. Neural Netw. Learn. Syst. 2018, 29, 2192-2203. [CrossRef]

6. Yazdanian, M.; Mehrizi-Sani, A. Distributed control techniques in microgrids. IEEE Trans. Smart Grid 2014, 5, 2901-2909. [CrossRef]

7. Tsianos, K.I.; Lawlor, S.; Rabbat, M.G. Consensus-based distributed optimization: Practical issues and applications in large-scale machine learning. In Proceedings of the 2012 50th Annual Allerton Conference on Communication, Control, and Computing, Allerton, Monticello, IL, USA, 1-5 October 2012.

8. Zhang, Z.; Chow, M.Y. Convergence analysis of the incremental cost consensus algorithm under different communication network topologies in a smart grid. IEEE Trans. Power Syst. 2012, 27, 1761-1768. [CrossRef]

9. Yang, S.; Tan, S.; Xu, J.X. Consensus based approach for economic dispatch problem in a smart grid. IEEE Trans. Power Syst. 2013, 28, 4416-4426. [CrossRef]

10. Yang, Z.; Xiang, J.; Li, Y. Distributed virtual incremental cost consensus algorithm for economic dispatch in a microgrid. IEEE Int. Conf. Control Autom. ICCA 2016, 2016, 383-388.

11. Lee, S.J.; Choi, J.Y.; Lee, H.J.; Won, D.J. Distributed coordination control strategy for a multi-microgrid based on a consensus algorithm. Energies 2017, 10, 1017. [CrossRef]

12. Cattivelli, F.S.; Sayed, A.H. Diffusion LMS strategies for distributed estimation. IEEE Trans. Signal Process. 2010, 58, 1035-1048. [CrossRef]

13. Yoo, H.J.; Nguyen, T.T.; Kim, H.M. Diffusion-Based Distributed Coordination Control of Power Converters in MG for Efficiency Improvement. IEEE Access 2019, 7, 53347-53357. [CrossRef]

14. Chen, J.; Sayed, A.H. Diffusion adaptation strategies for distributed optimization and learning over networks. IEEE Trans. Signal Process. 2012, 60, 4289-4305. [CrossRef]

15. Bui, V.H.; Hussain, A.; Kim, H.M. Diffusion strategy-based distributed operation of microgrids using multiagent system. Energies 2017, 10, 903. [CrossRef]

16. He, Y.; Wang, W.; Wu, X. Multi-Agent Based Fully Distributed Economic Dispatch in Microgrid Using Exact Diffusion Strategy. IEEE Access 2020, 8, 7020-7031. [CrossRef]

17. De Azevedo, R.; Cintuglu, M.H.; Ma, T.; Mohammed, O.A. Multiagent-Based Optimal Microgrid Control Using Fully Distributed Diffusion Strategy. IEEE Trans. Smart Grid 2017, 8, 1997-2008. [CrossRef]

18. Guo, F.; Wen, C.; Mao, J.; Song, Y.D. Distributed Secondary Voltage and Frequency Restoration Control of Droop-Controlled Inverter-Based Microgrids. IEEE Trans. Ind. Electron. 2015, 62, 4355-4364. [CrossRef]

19. Ahumada, C.; Cárdenas, R.; Sáez, D.; Guerrero, J.M. Secondary Control Strategies for Frequency Restoration in Islanded Microgrids With Consideration of Communication Delays. IEEE Trans. Smart Grid 2016, 7, 1430-1441. [CrossRef]

20. Simpson-Porco, J.W.; Shafiee, Q.; Dorfler, F.; Vasquez, J.C.; Guerrero, J.M.; Bullo, F. Secondary Frequency and Voltage Control of Islanded Microgrids via Distributed Averaging. IEEE Trans. Ind. Electron. 2015, 62, 7025-7038. [CrossRef]

21. Dehkordi, N.M.; Sadati, N.; Hamzeh, M. Distributed Robust Finite-Time Secondary Voltage and Frequency Control of Islanded Microgrids. IEEE Trans. Power Syst. 2017, 32, 3648-3659. [CrossRef] 
22. Cady, S.T.; Domínguez-García, A.D.; Hadjicostis, C.N. Finite-time approximate consensus and its application to distributed frequency regulation in islanded AC microgrids. Proc. Annu. Hawaii Int. Conf. Syst. Sci. 2015, 2015, 2664-2670.

23. Fu, R.; Wu, Y.; Wang, H.; Xie, J. A distributed control strategy for frequency regulation in smart grids based on the consensus protocol. Energies 2015, 8, 7930-7944. [CrossRef]

24. Wu, D.; Dragicevic, T.; Vasquez, J.C.; Guerrero, J.M.; Guan, Y. Secondary coordinated control of islanded microgrids based on consensus algorithms. In Proceedings of the 2014 IEEE Energy Conversion Congress and Exposition (ECCE), Pittsburgh, PA, USA, 14-18 September 2014; pp. 4290-4297.

25. Nguyen, C.K.; Nguyen, T.T.; Yoo, H.J.; Kim, H.M. Consensus-based SOC balancing of battery energy storage systems in wind farm. Energies 2018, 11, 3507. [CrossRef]

26. Chen, F.; Chen, M.; Li, Q.; Meng, K.; Zheng, Y.; Guerrero, J.M.; Abbott, D. Cost-Based Droop Schemes for Economic Dispatch in Islanded Microgrids. IEEE Trans. Smart Grid 2017, 8, 63-74. [CrossRef]

27. Chen, G.; Lewis, F.L.; Feng, E.N.; Song, Y. Distributed Optimal Active Power Control of Multiple Generation Systems. IEEE Trans. Ind. Electron. 2015, 62, 7079-7090. [CrossRef]

28. Hu, Y.F.; Blake, R.J. An improved diffusion algorithm for dynamic load balancing. Parallel Comput. 1999, 25, 417-444. [CrossRef]

29. Pogaku, N.; Prodanović, M.; Green, T.C. Modeling, analysis and testing of autonomous operation of an inverter-based microgrid. IEEE Trans. Power Electron. 2007, 22, 613-625. [CrossRef]

30. Wei, B.; Gui, Y.; Trujillo, S.; Guerrero, J.M.; Vasquez, J.C. Distributed Average Integral Secondary Control for Modular UPS Systems-Based Microgrids. IEEE Trans. Power Electron. 2019, 34, 6922-6936. [CrossRef]

31. Gui, Y.; Wei, B.; Li, M.; Guerrero, J.M.; Vasquez, J.C. Passivity-based coordinated control for islanded AC microgrid. Appl. Energy 2018, 229, 551-561. [CrossRef]

(C) 2020 by the authors. Licensee MDPI, Basel, Switzerland. This article is an open access article distributed under the terms and conditions of the Creative Commons Attribution (CC BY) license (http://creativecommons.org/licenses/by/4.0/). 